\title{
PREVENÇÃO DE ACIDENTES NA INFÂNCIA: ATUAÇÃO DO ENFERMEIRO NA ATENÇÃO PRIMÁRIA
}

\author{
PREVENTION OF ACCIDENTS IN CHILDHOOD: NURSE'S \\ PERFORMANCE IN PRIMARY CARE
}

Camylla Nunes Truta ${ }^{1}$

\begin{abstract}
RESUMO: Os acidentes na infância já se constituem como um problema de saúde pública, pois apresentam grande representação como causa de morbimortalidade. Eles podem ser controlados, uma vez que existe uma série de fatores que tornam sua ocorrência previsível. A sua prevenção significa garantir um espaço rico em oportunidades e seguro para a criança. O trabalho teve como objetivo geral elaborar uma proposta de intervenção para atuação do enfermeiro da Estratégia de Saúde da Família na prevenção de acidentes nos dois primeiros anos de vida. Trata-se de uma pesquisa do tipo Revisão Integrativa baseada em pesquisas com temáticas similares, sendo, portanto um importante instrumento para a Prática Baseada em Evidências (PBE). Dentre os tipos de acidentes que mais acometem as crianças até os dois anos de idade, encontram-se as quedas, cortes, queimaduras, contusões e a sufocação. Tendo em vista que a formação do enfermeiro da saúde coletiva está voltada à prevenção e promoção da saúde, estes profissionais se encontram capacitados a desenvolver estas ações visando a prevenção de acidentes na infância. Ao analisar os estudos pode-se realizar um planejamento para desenvolver programas educacionais de prevenção de acidentes e atuar nos espaços que permitam essa comunicação seja com pais, professores e até mesmo a comunicação em massa através de sites e mídias televisivas. Com isto, visa-se diminuir o número de acidentes na infância causados pela falta de atenção aos infantes e ao ambiente em que eles convivem.
\end{abstract}

Palavras chave: Enfermeiros. Estratégia Saúde da Família. Prevenção de Acidentes.

ABSTRACT: Childhood accidents are already a public health problem, as they are highly represented as a cause of morbidity and mortality. They can be controlled, as there are a number of factors that make their occurrence predictable. Its prevention

\footnotetext{
${ }^{1}$ Enfermeira da Estratégia Saúde da Família. Especialista em Saúde da Família com Ênfase na Atenção Primária - FIP, Bacharelado em Enfermagem - UFCG.
} 
means guaranteeing a space rich in opportunities and safe for the child. The general objective of the work was to elaborate an intervention proposal for the nurses' actions in the Family Health Strategy to prevent accidents in the first two years of life. It is an Integrative Review type research based on research with similar themes, and is therefore an important tool for Evidence-Based Practice (EBP). Among the types of accidents that most affect children up to two years of age, are falls, cuts, burns, bruises and suffocation. Bearing in mind that the training of public health nurses is focused on prevention and health promotion, these professionals are able to develop these actions aimed at preventing accidents in childhood. When analyzing the studies, planning can be carried out to develop educational accident prevention programs and to act in spaces that allow this communication, whether with parents, teachers and even mass communication through websites and television media. With this, the aim is to reduce the number of childhood accidents caused by the lack of attention to infants and the environment in which they live.

Keywords: Nurses. Health Strategy. Accidents prevention. 


\section{INTRODUÇÃO}

Os acidentes na infância já se constituem como um problema de saúde pública, pois apresentam grande representação como causa de morbimortalidade. $O$ alto índice de acidentes nessa fase requer a existência de ações preventivas junto à família, criança, profissional da saúde e sociedade, buscando uma melhor qualidade de vida para os infantes (AMARAL, et al.,2009).

Dados obtidos através da página Criança Segura (2020), apontam para um número alarmante de acidentes a infância que chegam à hospitalização e até a morte. Foram, no ano de 2018 (dado mais atualizado da página), cerca de 13.000 registros de quedas e 7.000 registros de queimaduras em crianças menores de quatro anos. No ano de 2017, cerca de 1.700 mortes foram registradas nessa faixa etária em decorrência de acidentes de trânsito e domésticos.

Bruno et al. (2006), apontam que a infância é uma fase marcada por crescimento e desenvolvimento ativos, caracterizando-se como um período em que as crianças são expostas a riscos ambientais motivadas pela curiosidade aumentada e pouco discernimento acerca das situações de perigo à vida.

Nessa fase da vida as crianças são expostas a diversos ambientes e correm riscos que se tornam maiores quando levados em conta a sua incapacidade de proteger-se. Assim, observa-se a cada dia nos noticiários o relato comum de acidentes envolvendo as crianças, estes vão desde eventos menos graves que não deixam sequelas físicas até outros fatais.

No entanto, os acidentes podem ser controlados, uma vez que existe uma série de fatores que tornam sua ocorrência previsível. A prevenção de acidentes significa garantir um espaço rico em oportunidades e seguro para que a criança, em qualquer faixa etária, possa adquirir novas competências e ampliar a rede de interações e adaptação a novos espaços e rotinas (COELHO; SILVA, 2011).

A criança para desenvolver-se precisa experimentar ambientes, situações, brincadeiras e experiências novas, as quais não está acostumada, estimulando 
assim o seu desenvolvimento intelectual pleno. Para que isso ocorra, os pais, professores e responsáveis precisam estar atentos e ofertar este conjunto de fatores benéficos de forma segura. Os ambientes em que elas vivem precisam estar livres de riscos, para isto se faz importante a prevenção de acidentes na infância.

Pais, professores e a sociedade em geral muitas vezes encontram-se limitados a evitar os acidentes na infância por não conhecer os riscos que se apresentam no ambiente, nos alimentos, na socialização das crianças, nas brincadeiras de cada faixa etária e até mesmo nas características pessoais das crianças.

Nesse contexto, entende-se que os enfermeiros da Estratégia de Saúde da Família (ESF), durante as consultas de puericultura e as atividades do Programa Saúde na Escola (PSE), necessitam de conhecimentos diversos acerca das fases de crescimento e desenvolvimento infantis para que possam atuar de forma integral e holística no cuidado à saúde das crianças, promovendo saúde a partir da prevenção de acidentes, uma vez que esses quando acometem as crianças podem lhes causar danos.

A partir da elaboração de materiais teóricos abordando a temática, acadêmicos e profissionais da saúde podem desenvolver programas de prevenção de acidentes seja na atenção primária, nas creches e escolas, nos prontoatendimentos e hospitais infantis. Nestes cenários o enfermeiro apresenta condições para desenvolver suas habilidades como educador em saúde, podendo desenvolver estas mudanças ou mesmo capacitar a população na mudança de atitudes com relação à prevenção.

O trabalho teve como objetivo geral elaborar uma proposta de intervenção para atuação do enfermeiro da ESF na prevenção de acidentes nos dois primeiros anos de vida. Para tanto, os objetivos específicos foram realizar uma revisão integrativa sobre prevalência de acidentes na infância; conhecer o papel do enfermeiro frente a esta problemática na literatura investigada; e descrever ações do enfermeiro na ESF para prevenção de acidentes na infância. 


\section{METODOLOGIA}

Trata-se de uma pesquisa do tipo Revisão Integrativa, baseada em pesquisas com temáticas similares, sendo esta uma metodologia que sintetiza conhecimentos já divulgados incorporando aplicabilidade à estes, sendo portanto um importante instrumento para a Prática Baseada em Evidências (PBE), esta por sua vez vem aprimorando e enriquecendo a ciência da enfermagem, auxiliando os profissionais nas tomadas de decisões clínicas.

Foram utilizados como critérios para a inclusão nesta pesquisa: Artigos cuja tipologia metodológica envolvesse fundamentos quantitativos e/ou qualitativos, experimentais, observacionais, de coorte e transversais, estudo de caso e documentais; publicados em língua portuguesa, no período compreendido entre 2000 e 2019, que encontravam-se disponíveis nos bancos de dados em versão completa com livre acesso ao público.

Para melhor avaliação dos artigos foi realizada leitura prévia dos resumos para verificar real adequação aos objetivos desta pesquisa. Após a seleção dos artigos científicos ocorreu a leitura criteriosa dos textos e análise dos resultados das pesquisas. Em seguida, os trabalhos foram reduzidos a fichas com conteúdo relevante para esta pesquisa. Durante todas as etapas analíticas foram confeccionados fichamentos para uma melhor organização e compreensão dos resultados, sendo estes categorizados baseando-se no método de revisão integrativa. 


\section{RESULTADOS E DISCUSSÃO}

\section{Caracterização dos tipos de acidentes que mais acometem as crianças até os dois anos de idade}

No Brasil, os acidentes de trânsito e os afogamentos são as principais causas de mortalidade, seguidos por sufocações, queimaduras, quedas e intoxicações. (CRIANÇA SEGURA BRASIL, 2019).

Dados complementares acerca dos acidentes ocorridos com crianças apontam que a maioria dos acidentes ocorrem no domicílio, no período da tarde (GONÇALVES et al., 2019). Outro estudo brasileiro reforça que a maior parte dos acidentes ocorreu no domicilio e refere que a locomoção para o hospital foi mais freqüente em veículo particular, tendo os acidentes predominado no período diurno, durante a semana (MALTA, 2015).

Estudo publicado por Barcelos (2017) traz importantes relações acerca das condições sociais da família e a ocorrência dos acidentes. O autor destaca que os fatores como mãe adolescente, baixa escolaridade materna e baixa renda familiar estiveram associados ao maior índice de quedas, cortes e queimaduras. O estudo ainda apresenta que a incidência de quedas e queimaduras triplica e a de quedas duplica no segundo ano de vida, em comparação ao primeiro.

Em se tratando das quedas, Martins e Andrade (2010) apontam que em sua maioria são de baixa gravidade, levando as crianças à internação ou mesmo ao atendimento ambulatorial. No entanto, neste mesmo estudo eles destacam a ocorrência de um óbito por traumatismo intracraniano decorrente de queda em uma criança, este fato, ainda que isolado no estudo, aponta para importância de se prevenir esse tipo de acidente na infância. Esses autores afirmam que a ocorrência do traumatismo craniano em crianças pode estar relacionada à ausência ou diminuição dos reflexos de proteção ainda não desenvolvidos nestes. 
Malta (2015) destaca que as contusões foram as lesões mais frequentes, atingindo principalmente as regiões de cabeça e pescoço. Este estudo indica que as quedas foram as ocorrências que levaram à maior proporção de atendimentos nos serviços de urgência e emergência. Outro estudo, publicado por Gonçalves et al. (2019), confirma que as regiões do corpo que sofreram mais lesões após as quedas foram a cabeça, face e pescoço, sendo as lesões do segmento cefálico mais frequentes em crianças mais jovens. Barcelos (2017) ainda reforça que no primeiro ano de vida, cerca de metade das crianças sofreu, pelo menos, um episódio de queda.

Os cortes e contusões geralmente decorrem das quedas e tem sua gravidade relacionada à região do corpo atingida, lesões que atingem a cabeça e o tronco apresentam maior gravidade devido à localização dos órgãos vitais como cérebro, coração, pulmão, rins e fígado (MALTA et al, 2012).

Estudos acerca das queimaduras na infância como os de Montes et al. (2011), Batista et al. (2011) e Millan et al. (2012) revelam que estas geralmente são causadas por escaldadura e combustão de álcool. As vítimas de queimaduras que atingem grande parte do corpo geralmente são submetidos a procedimentos invasivos como a sondagem vesical de demora, podendo ainda se submeter a intervenções cirúrgicas como o enxerto e o desbridamento, todos estes procedimentos colocam o queimado em risco de infecção o que pode agravar ainda mais seu quadro clínico.

Grandes queimados estão ainda susceptíveis a presença de alterações na temperatura corporal devido a perda da integridade tissular em grande extensão e alterações metabólicas decorrentes da situação. São comuns episódios de ansiedade relacionada a dor e ao afastamento do lar, este segundo mais presente nos casos de internação hospitalar. A circulação sanguínea também é alterada devido o comprometimento dos vasos sanguíneos que irrigam a região afetada pela queimadura, gerando quase sempre o edema e também problemas na amplitude dos movimentos quando a lesão atinge articulações (BATISTA,et al., 2011).

Pesquisa norte-americana divulgada pelo site brasileiro Atlas da Saúde (2013) afirmou que o número de casos de crianças que sofrem asfixia acidental (sufocação) tem aumentado. Estes casos decorrem em sua maioria da ingestão de alimentos tais 
como carnes, ossos e frutos secos de cascas rígidas. Mesmo o estudo apresentando uma alta taxa de crianças que receberam alta após o atendimento, sabe-se que a asfixia pode ser fatal ou deixar sequelas graves na criança devido ao bloqueio das vias respiratórias das crianças e à consequente ausência de ventilação pulmonar durante o episódio de asfixia.

\section{Enfermeiro da atenção básica como educador em saúde}

É preciso entender como a enfermagem tem importante papel na temática dos acidentes na infância. Para iniciar a discussão traz-se a ideia de Gomes (2004) de que na enfermagem o cuidar da criança significa prestar assistência integral e individualizada visando desenvolver ao máximo seu potencial, prevenir intercorrências e proporcionar um viver feliz e saudável. Dentro dessa concepção, a prevenção de acidentes destaca-se visto que enquanto se previne acidentes na infância, evita-se que a criança desenvolva problemas de saúde.

Em estudo realizado por Gonçalves et al. (2019), afirma-se que cerca de 90\% das lesões não intencionais podem ser evitadas por meio de medidas de prevenção, sendo comprovada a redução nos índices de acidentes após implantação dessas medidas.

Martins (2006) afirma que a prevenção é o caminho mais eficaz para reduzir os índices elevados de acidentes na infância, essa prevenção pode ocorrer a partir de programas educacionais desde a pré-escola e junto à comunidade, além do cumprimento de normas e medidas de proteção junto à indústria farmacêutica e engenharia civil. Além desses fatores é importante conhecer a natureza e a realidade acerca dos acidentes na infância para formar um diagnóstico que auxilie na elaboração e implementação das estratégias específicas de prevenção em cada público.

A partir desta afirmação percebe-se que a prevenção de acidentes na infância está relacionada a diversos setores seja o de saúde, educação, comunicação, esportes, indústria alimentícia, de medicamento, de produtos de limpeza, entre 
outros. Portanto, diversos profissionais podem e devem se engajar em programas que tenham como objetivo difundir as informações acerca da prevenção de acidentes e também de proteção às crianças e prevenção de acidentes.

A Sociedade Brasileira de Enfermeiros Pediatras fez uma convocação para a classe enfatizando que o enfermeiro tem, junto à sociedade, a responsabilidade por iniciar e apoiar ações que satisfaçam as necessidades de saúde dos mesmos, devendo se valer de todas as oportunidades de intervenções que promovam a adoção de medidas de segurança e mudanças de comportamento, com a finalidade de ter um mundo mais seguro para as crianças. (BELELO-ANACLETO e MANDETTA, 2016).

Sabendo disso, tem-se ainda o conceito de que o enfermeiro além de cuidador é um educador por natureza, visto que cuidado e educação são ideologias inseparáveis. O profissional enfermeiro "tem em sua formação a essência do cuidado ao ser humano de forma holística, visando, principalmente, a promoção da saúde, que envolve as condições de trabalho, de moradia, de alimentação, do meio ambiente e de lazer" (MOTTA et al., 2012, p. 772).

Além disso, o enfermeiro é o profissional capacitado para compreender as peculiaridades do crescimento e desenvolvimento da criança (MOTTA, et al., 2012). Por isso, sua inserção é tão importante no contexto da prevenção de acidentes, uma vez que compreendendo o crescimento e desenvolvimento ele poderá atuar classificando os principais acidentes de acordo com a faixa etária da criança.

Autores como Neto et al. (2010) ressaltam que a Estratégia Saúde da Família (ESF) tem a responsabilidade de trabalhar junto à comunidade e os setores sociais, dessa forma o enfermeiro da ESF pode atuar na prevenção e orientação quanto aos riscos de acidentes que podem ser preveníveis. A atuação do enfermeiro da ESF não está restrita à unidade de saúde e dessa forma sua possibilidade de atingir públicos maiores e diversos aumenta.

Para Alves e Silva (2003), é importante que os enfermeiros durante suas consultas às crianças incluam orientações à família com relação a supervisão das brincadeiras, inspeção dos brinquedos novos e usados, manutenção, limpeza e armazenamento dos brinquedos em local seguro e segurança do ambiente onde as crianças ficam e interagem. Portanto, estando engajado nos programas de 
prevenção de acidentes na infância, o enfermeiro desenvolve sua função profissional de cuidar de forma holística da saúde da população, em especial das crianças contribuindo para um crescimento e desenvolvimento saudáveis.

Tendo em vista que a formação do enfermeiro da saúde coletiva está voltada à prevenção e promoção da saúde, estes profissionais se encontram capacitados a desenvolver estas ações visando a prevenção de acidentes na infância. Batista et al. (2011), destacam que cabe ao enfermeiro inserido na atenção básica o papel de orientador dos adultos quanto às medidas de prevenção de acidentes na infância, este objetivo pode ser atingido a partir da orientação da população por meio de cartazes, palestras, oficinas, além do espaço para educação individualizada nas consultas de puericultura.

\section{Plano de Ação para atuação do enfermeiro na ESF}

Através dos marcos no desenvolvimento presentes nas cadernetas individuais de acompanhamento das crianças e relacionando estes marcos aos acidentes infantis pode-se perceber como períodos de maior atenção as seguintes idades: aos 4 meses, a criança alcança e pega objetos pequenos; aos 9 meses, arrasta-se ou engatinha; aos 18 meses, a criança anda sozinha e retira qualquer peça do vestuário; e aos 2 anos, corre e/ou sobe degraus baixos.

Vale ressaltar que se faz necessário a supervisão contínua de adultos, pais ou responsáveis, para atentar-se aos riscos do ambiente, sem prejudicar o desenvolvimento das crianças, estimulando as descobertas e buscando sempre protegê-las. (MALTA et al., 2015).

Além do mais, estudos já apontam que é necessária a supervisão constante dos riscos ambientais tais como, tapetes soltos, pisos molhados, móveis com quinas, vidros, janelas sem proteção, berços sem proteção, camas elevadas, brinquedos espalhados, brinquedos pequenos que podem ser introduzidos como corpo estranho, objetos perfurocortantes, fogão, medicamentos e produtos de limpeza mal armazenados, animais domésticos (MARTINS, 2006; PAES, GASPAR, 2005). 
A partir dessas informações é descrito abaixo uma proposta de intervenção a ser desenvolvida em comunidades atendidas pela ESF, no qual o enfermeiro atua como peça fundamental na educação em saúde voltada à prevenção dos acidentes na infância.

- Intervenção 1: Orientações às gestantes

- A partir da trigésima semana de gestação abordar os temas descritos no Quadro 1, em Rodas de Conversa na própria Unidade de Saúde:

QUADRO 1 - ORIENTAÇÕES ÀS GESTANTES.

Temas abordados

Risco prevenido

Transporte do Recém Nascido (Uso de assentos de Risco de lesão por segurança em automóveis desde o primeiro dia de vida) acidente de trânsito

Local, posicionamento e cuidados durante o sono do bebê (Evitar que o bebê durma no mesmo leito que os pais; posição "de barriga para cima" no leito; e não colocação

Risco de asfixia de lençóis e almofadas próximos do bebê)

Segurança do bebê (Nunca deixar uma criança sozinha em locais como sofás, trocadores, camas e etc. assim como sob a responsabilidade de crianças pequenas)

Atenção no manuseio de alimentos e no preparo do banho do recém-nascido (contato líquidos e objetos com temperatura elevada)

Risco de quedas

Risco de

queimaduras

- Intervenção 2: Orientações aos pais

- Orientar os pais nas consultas de puericultura de acordo com a idade da criança na própria Unidade de Saúde atentando para os marcos de desenvolvimento como descritos no Quadro 2: 
QUADRO 2: ORIENTAÇÕES AOS PAIS.

\begin{tabular}{|c|c|}
\hline Idade & Marco do Desenvolvimento/Temas abordados \\
\hline 4 meses & $\begin{array}{l}\text { Alcança e pega objetos pequenos: atentar os pais paraRisco de } \\
\text { possibilidade de alcance de objetos próximos a ela,intoxicação } \\
\text { assim como rolamentos na cama ou outros locais emquedas } \\
\text { que for colocada }\end{array}$ \\
\hline 9 meses & $\begin{array}{l}\text { Arrasta-se ou engatinha: Orientar que seja redobada osMaior } \\
\text { cuidados com o ambiente, uma vez que a capacidadequedas } \\
\begin{array}{lc}\text { de locomover-se passa a aumentar } & \text { intoxicações }\end{array}\end{array}$ \\
\hline 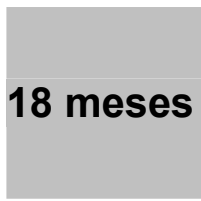 & $\begin{array}{l}\text { Anda sozinha e tira qualquer peça do vestuário: ExporRisco de quedas } \\
\text { que a criança passa a utilizar de sua independência,asfixia } \\
\text { mas o controle motor é novo e a criança ainda não está } \\
\text { totalmente adaptada podendo se desequilibrar }\end{array}$ \\
\hline an & $\begin{array}{l}\text { Corre elou sobe degraus baixos: Explicar que nesseRisco de quedas de } \\
\text { momento formam-se as noções de distância e demaiores alturas } \\
\text { profundidade, não sendo esta habilidade totalmente } \\
\text { dominada pela criança }\end{array}$ \\
\hline
\end{tabular}

- Intervenção 3: Treinamento de primeiros socorros na comunidade.

- Buscar na comunidade pais, responsáveis, líderes comunitários e professores que demonstrem interesse no treinamento de Primeiros Socorros.

- Abordar os temas que mais acometem as crianças como verificado nesta revisão: queda, corte, fratura e torção, engasgo/asfixia, intoxicação e afogamento.

- Utilizar sempre materiais atualizados.

\section{CONSIDERAÇÕES FINAIS}

É importante destacar que para atuação na prevenção de acidentes na infância através de programas é necessário inicialmente conhecer as características envolvidas nestes acidentes para que o foco seja definido e as medidas possam surtir efeitos positivos na saúde da criança. Este estudo concluiu que os tipos de acidentes que mais acometem as crianças até os dois anos de idade são as quedas, cortes, queimaduras, contusões e a asfixia. 
Através dos marcos no desenvolvimento das crianças e relacionando estes marcos aos acidentes infantis pode-se perceber como períodos de maior atenção aos infantes as faixas etárias de quatro, nove e dezoito meses e aos dois anos.

Salienta-se aqui a importância de que os responsáveis estejam atentos a estes riscos e protejam o ambiente, seja doméstico, escolar ou mesmo o espaço urbano, para que as crianças possam se desenvolver e crescer livres de danos a sua saúde, em especial aqueles decorrentes de acidentes, sabendo-se que estes são evitáveis e preveníveis.

Ao analisar os estudos pode-se realizar um planejamento para desenvolver programas educacionais de prevenção de acidentes e atuar nos espaços que permitam essa comunicação seja com pais, professores e até mesmo a comunicação em massa através de sites e mídias televisivas. Com isto, visa-se diminuir o número de acidentes na infância causados pela falta de atenção aos infantes e ao ambiente em que eles convivem.

O enfermeiro de saúde coletiva está voltado à prevenção e promoção da saúde e, por isso, esses profissionais se encontram capacitados a desenvolver ações visando a prevenção de acidentes na infância. As ações podem ser desenvolvidas a partir da orientação da população por meio de cartazes, palestras, oficinas, além do espaço para educação individualizada nas consultas de puericultura. 


\section{REFERÊNCIAS BIBLIOGRÁFICAS}

ALVES, A. M. A.; SILVA, L. R. Brincadeiras na infância: crescimento e saúde. In: FIGUEIREDO, N.M.A. [Org.] Ensinando a cuidar da criança. - São Caetano do Sul, SP: Difusão Enfermagem, 2003.

AMARAL, E.M.S.; SILVA, C.L.M. da; PEREIRA, E.R.R.; GUARNIERI, G.; BRITO, G. S.S.de; OLIVEIRA, L.M.de. Incidência de acidentes com crianças em um pronto-socorro infantil. RevinstCiênc Saúde, v. 27, n. 4, p.313-7, 2009.

ATLAS da Saúde. Aumenta número de casos de asfixia acidental de crianças. 31 jul., 2013. 16h26min. Disponível em: <http://www.atlasdasaude.pt/publico/content/aumenta-numero-decasos-de-asfixia-acidental-de-criancas>. Acesso em: 06 de fev. de 2014.

BARCELOS, R.S.; SANTOS, I.S.; MATIJASEVICH, A.; BARROS, A.J.D.; BARROS, F.C.; FRANÇA, G.V.A.; SILVA, V.L.S. Acidentes por quedas, cortes e queimaduras em crianças de 04 anos: coorte de nascimentos de Pelotas, Rio Grande do Sul, Brasil, 2004. Cad. Saúde Pública, v. 33, n. 2, 2017.

BATISTA, L.T. O.; RODRIGUES, F.A.; VASCONCELOS, J. M.B. Características clínicas e diagnósticos de enfermagem em crianças vítimas de queimadura. Rev Rene, Fortaleza, v. 12, n. 1, p.158-65, jan/mar. 2011.

BELELA-ANACLETO, A.S.C; MANDETTA, M.A. Prevenção de acidentes na infância: uma convocação da "Sociedade Brasileira de Enfermeiros Pediatras", 2016. Disponível em: <http://dx.doi.org/10.1590/1982-0194201600068>. Acesso em: 20 de fevereiro de 2020.

BRUNO, B.B.; FONSECA, M.C.; DIAS, T.P.; MOREIRA, L.R.C. Prevenção de Acidentes com crianças de 3 a 6 anos de idade em uma creche no município de São José dos Campos. Anais... X Encontro Latino Americano de Iniciação Científica e VI Encontro Latino Americano de Pós-graduação - Universidade do Vale do Paraíba. 2006.

COELHO, L.C.A.; SILVA, L.R.C.da. Formação docente, educação infantil e prevenção de acidentes. X Congresso Nacional de Educação - EDUCERE. Pontifícia Universidade Católica do Paraná, Curitiba, 7 de nov. 2011.

CRIANÇA Segura Brasil [Internet]. Os acidentes em números: conheça os dados sobre acidentes. São Paulo (SP): Criança Segura Brasil. 2019. Disponível em: <https://criancasegura. org.br/dados-de-acidentes/>. Acesso em: 01 de março de 2020.

GOMES, V.L. de O. A interpretação do cuidado de Enfermagem à crianças em creches, pela ótica de Pierre Bourdieu. Florianópolis: UFSC/PEN. 2004. 197 p. (Tese de doutorado Programa de Pós-graduação em Enfermagem - Universidade Federal de Santa Catarina, 2004).

GONÇALVES, A.C.; ARAÚJO, M.P.B.; PAIVA, K.V.; MENEZES, C.S.A.; SILVA, A.É.M.C.; SANTANA, G. O. ; ORTOLAN, É.V.P.; LOURENÇÃO, P.L.T.A. Acidentes na infância: casuística de um serviço terciário em uma cidade de médio porte do Brasil. Rev Col Bras Cir, v. 46, n. 2 , 2019.

MALTA, D.C.; MASCARENHAS, M.D.M.; BERNAL, R.T.I.; VIEGAS, A.P.B.; SÁ, N. N.B.; SILVA JUNIOR, J.B. Acidentes e violência na infância: evidências do inquérito sobre atendimentos de emergência por causas externas - Brasil, 2009. Ciência \& Saúde Coletiva, v. 17, n. 9, p.22472258, 2012.

MALTA, D.C,; MASCARENHAS, M.D.M.; NEVES, A.C.M.; SILVA, M.A. Atendimentos por 
acidentes e violências na infância em serviços de emergências públicas. Cad. Saúde Pública, Rio de Janeiro, v. 31, n. 5, p. 1095-1105, mai, 2015.

MARTINS, C.B.de G. Acidentes na infância e adolescência: uma revisão bibliográfica. RevBrasEnferm, v. 59, n. 3, p. 344-8, mai-jun, 2006.

MARTINS, C.B. de G.; ANDRADE, S.M. Estudo descritivo de quedas entre menores de 15 anos no município de Londrina (PR, Brasil). Ciência \& Saúde Coletiva, v. 15, n. 2, p.3167- 3173, 2010.

MILLAN, L.S.; GEMPERLI, R.; TOVO, F.M.; MENDAÇOLLI, T.J.; GOMEZ, D.S.; FERREIRA, M.C. Estudo epidemiológico de queimaduras em crianças atendidas em hospital terciário na cidade de São Paulo. RevBrasCirPlást., v. 27, n. 4, p. 611-5, 2012.

MONTES, S.F.; BARBOSA, M.H.; SOUSA NETO, A.L. Aspectos clínicos e epidemiológicos de pacientes queimados internados em um Hospital de Ensino. RevEscEnferm USP, v. 45, n. 2, p. 369-73, 2011.

MOTTA, J.de A.; SILVA, P.O.da.; MARTA, C.B. ARAÚJO, B.B.M.de.; FRANCISCO, M.T.R.; JUNIOR, H.C.S. O cuidado à criança na creche: integração entre saúde e educação. Rev. enferm. UERJ, Rio de Janeiro, v. 20, esp.2, p.771-6, dez. 2012.

NETO, C.M.; ALVES, N.C.; PAES, M.S.L. Risco de acidentes na infância em uma creche comunitária de Ipatinga/MG. Revista Enfermagem Integrada - Ipatinga: Unileste-MG, v.3, n.1, Jul.-Ago. 2010.

PAES, C.E.N.; GASPAR, V.L.V. As injúrias não intencionais no ambiente domiciliar: a casa segura. J Pediatr, v. 81, p. 146-54, 2005. 